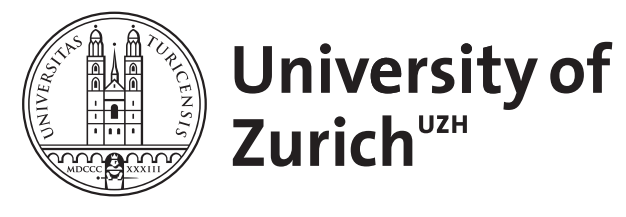

\title{
Operational calibration of APEX
}

Hueni, Andreas ; Sterckx, Sindy ; Jehle, Michael

\begin{abstract}
APEX system calibration has reached on operational level with calibration procedures at the CHB being well established and the APEX CAL IS enabling an efficient and transparent data storage and processing for the timely generation of calibration cubes required for the radiometric calibration of APEX imagery. Overall, these achievements result in shorter times from laboratory calibration to processed end user products.
\end{abstract}

DOI: https://doi.org/10.1109/IGARSS.2013.6723816

Posted at the Zurich Open Repository and Archive, University of Zurich

ZORA URL: https://doi.org/10.5167/uzh-92059

Conference or Workshop Item

Published Version

Originally published at:

Hueni, Andreas; Sterckx, Sindy; Jehle, Michael (2013). Operational calibration of APEX. In: Geoscience and Remote Sensing Symposium (IGARSS), 2013 IEEE International, Melbourne, Australia, 21 July 2013 - 26 July 2013. IEEE Xplore, 4423 -4426.

DOI: https://doi.org/10.1109/IGARSS.2013.6723816 


\title{
OPERATIONAL CALIBRATION OF APEX
}

\author{
A. Hueni ${ }^{1}$, S. Sterckx², M. Jehle ${ }^{1}$ \\ ${ }^{1}$ Remote Sensing Laboratories, University of Zurich, Winterthurerstrasse 190, 8057 Zurich, Switzerland \\ ${ }^{2}$ VITO, Boeretang 200, BE-2400 Mol, Belgium
}

\section{INTRODUCTION}

ESA's Airborne Imaging Spectrometer APEX (Airborne Prism Experiment) was developed under the PRODEX (PROgramme de Développement d'EXpériences scientifiques) program by a Swiss-Belgian consortium and entered its operational phase at the end of 2010. It features up to 532 spectral bands in full spectral mode while APEX spectral programmability enables achieving higher Signal-to-Noise-Ratios (SNR) by reducing the number of bands in a binned configuration. Data are acquired in 1000 pixels across track with a FOV of $28^{\circ}$ [1].

This paper describes the standard calibration of APEX at the calibration home base, the APEX calibration information system and the processing methods used for the generation of calibration cubes required for the level1 (radiance level) processing of APEX imaging cubes.

\section{METHODS}

\subsection{APEX Calibration}

APEX calibration is carried out at the CHB (Calibration Home Base) situated at DLR Oberpfaffenhofen [2]. The standard APEX calibration is carried out in an operational manner at least once a year, usually at the beginning of the flight season. The calibration comprises measurements on an optical bench for the geometric and spectral calibration, using a collimator-slit setup and a monochromator respectively, and radiometric calibration using a small and big integrating sphere for absolute radiometric calibration and flat-fielding respectively.

A full sensor calibration at the CHB can be achieved within 3-4 days, including sensor installation and alignment on the calibration bench.

Data volumes acquired during calibrations are largely governed by the spectral calibration; standard calibration data amount to $13 \mathrm{~GB}$ while special experiment carried out to enhance the system knowledge can reach around 16GB (Figure 1). The total expected raw data volume over the nominal lifetime of the system is an estimated 290-410 GB for two scenarios with constant or exponentially declining number of special experiments [3]. 


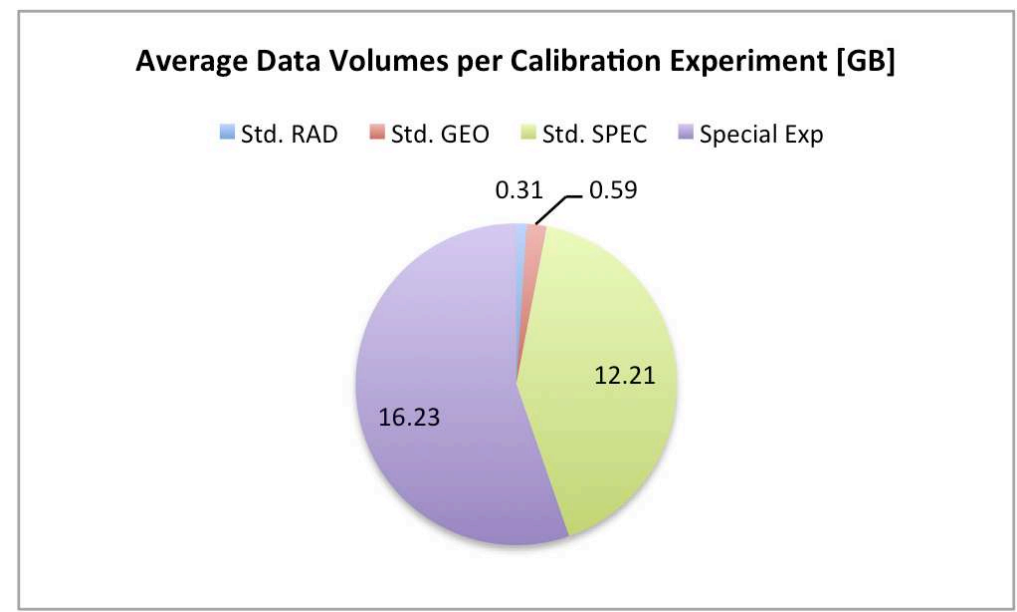

Figure 1: Average data volumes acquired during CHB missions per calibration experiment [GB]

\subsection{APEX Calibration Information System}

The APEX Calibration Information System (CAL IS) has been developed to deal with the large volumes of calibration related frames and associated metadata [3]. Most characteristic of the APEX calibration data is the large number of metaparameters per frame that depends upon the actual calibration step and ranges between 157217 entries per frame. The metadata describes both the CHB instrument configuration and the APEX system settings and various auxiliary sensor readings, such temperatures and pressures.

The APEX CAL IS consists of a relational database management system (RDBMS), a middleware written in Java and higher-level functions implemented in Java and Matlab.

The CAL IS currently comprises functions that allow the interactive radiometric and spectral coefficient determination from the data acquired in the CHB. Additional rich functionality is focused on the analysis of the system characteristics and allows interactive data exploration.

Geometric calibration and determination of the bad pixel map are still based on the Calibration Test Master software [4] at the time of writing, but are expected to be integrated into the CAL IS till mid 2013.

\subsection{Calibration Cube Generation}

Calibration cubes are required by the APEX PAF (Processing and Archiving Facility) [5] level-1 processing and comprise a number of layers holding calibration coefficients per spatio-spectral pixel: radiometric gains and offsets, across-track angles, centre wavelengths, FWHMs (Full Width at Half Maximum), bad pixels and vignetting.

These layers are generated by the APEX CAL IS and the CTM, applying interpolation routines to derive coefficients per pixel where sampling patterns were applied, as is the case for the spectral and geometric calibration. 


\section{RESULTS}

The APEX CAL IS has reached operational status and allows the generation of radiometric and spectral calibration layers in an automated fashion while giving the calibration scientist the tools to inspect each step of the information extraction as well as interactively explore both imaging frames and metadata. As the final calibration layers are held by the system as well, monitoring of the instrument performance over the years is extremely easy and allows an immediate assessment of the sensor status and success of the calibration procedures once new data have been entered into and processed by the CAL IS; in fact the system is capable of producing the full set of calibration layers before even leaving the calibration laboratory, giving the change to repeat flawed measurements almost immediately.

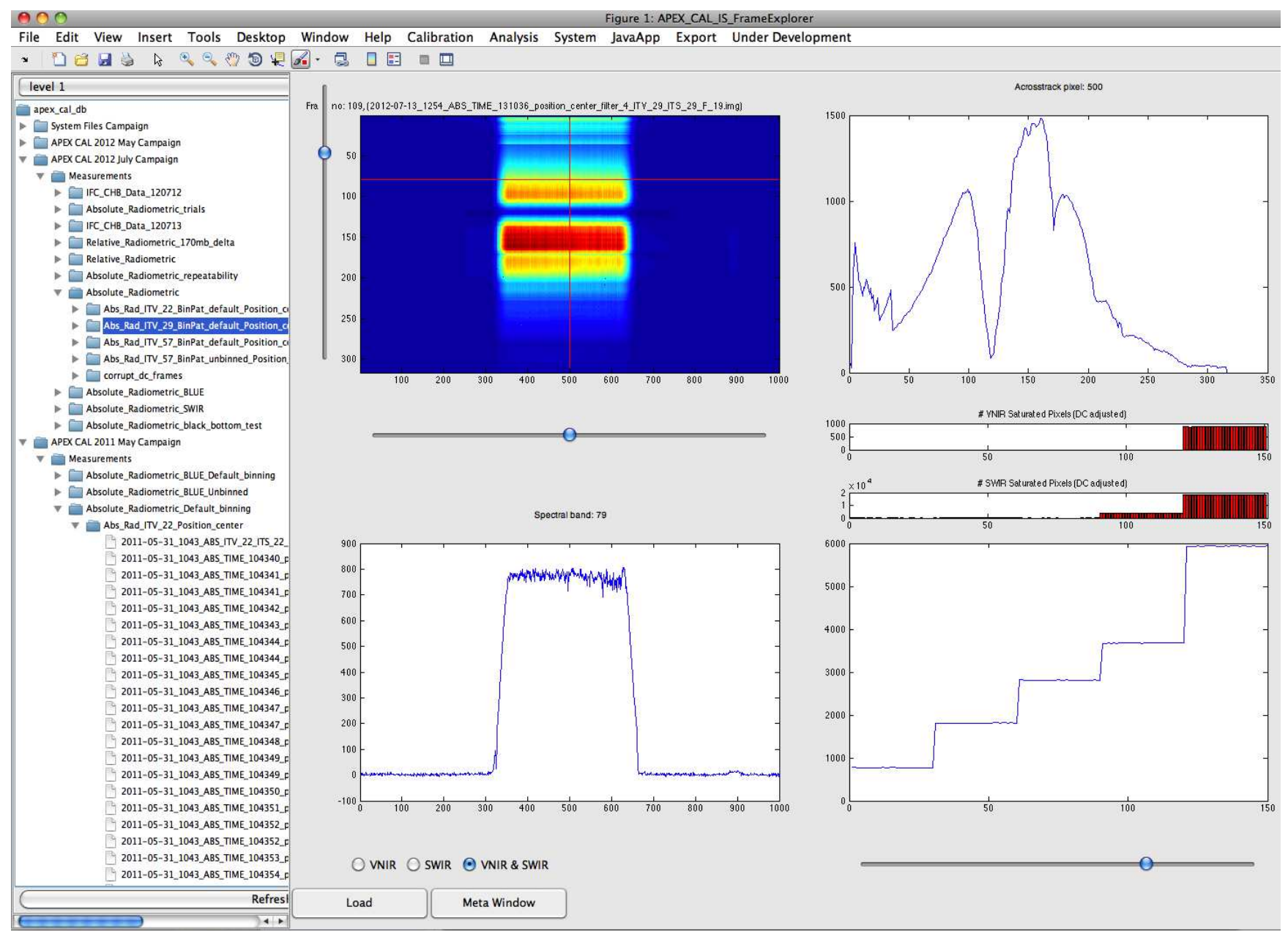

Figure 2: Matlab APEX CAL IS main interface

The main interface (Figure 2) is described hereafter: a hierarchical data browser component is featured on the left of the window, showing the data held by the database, organised by campaigns while the directory structure 
below each campaign reflects the original storage on disc. Selected data are visualised in the four displays to the right of the data browser, showing a frame view with 1000 pixels acrosstrack and spectral pixels depending on the binning pattern (top left), a spectral profile of the selected spatial position (top right), an acrosstrack profile for the selected spectral position (bottom left), and an alongtrack profile for the selected acrosstrack and spectral position (bottom right). Positions within this virtual cube are selected with three scrollbars placed at the edges of the frame display and alongtrack display respectively. Radio controls (bottom left) allow focusing the display on VNIR, SWIR or both detectors. Two smaller data displays (middle right) show the number of saturated pixels per frame of the virtual cube split into SWIR and VNIR detectors, basing on saturation data compiled during initial data loading. All further functions of the APEX CAL IS, such as calibration and analysis functions are accessed via menu entries provided by the main window.

The most data intensive process is the spectral calibration, mainly due to the involved data volumes and the complexity of the algorithms. The spectral layer generation time per detector exacts around 15-20 minutes, starting with dark current corrected frames as held by the APEX CAL IS database.

\section{CONCLUSIONS}

APEX system calibration has reached on operational level with calibration procedures at the CHB being well established and the APEX CAL IS enabling an efficient and transparent data storage and processing for the timely generation of calibration cubes required for the radiometric calibration of APEX imagery. Overall, these achievements result in shorter times from laboratory calibration to processed end user products.

\section{REFERENCES}

[1] M. E. Schaepman, M. Jehle, A. Hueni, K. Meuleman, The APEX Team, and K. I. Itten, "The 4th generation imaging spectrometer APEX and its application in Earth observation," IEEE Transactions on Geoscience and Remote Sensing, in preparation.

[2] P. Gege, J. Fries, P. Haschberger, P. Schötz, H. Schwarzer, P. Strobl, B. Suhr, G. Ulbrich, and W. J. Vreeling, "Calibration facility for airborne imaging spectrometers," ISPRS Journal of Photogrammetry \& Remote Sensing, vol. 64, pp. 387-397, 2009.

[3] A. Hueni, K. Lenhard, A. Baumgartner, and M. Schaepman, "The APEX (Airborne Prism Experiment Imaging Spectrometer) Calibration Information System," IEEE Transactions on Geoscience and Remote Sensing, in print.

[4] F. Dell'Endice, J. Nieke, D. Schlaepfer, A. Hueni, and K. Itten, "Automatic Calibration and Correction Scheme for APEX (Airborne Prism Experiment spectrometer)," in Proceedings of 5th EARSeL Workshop on Imaging Spectroscopy, Bruges, Belgium, 2007.

[5] A. Hueni, J. Biesemans, K. Meuleman, F. Dell'Endice, D. Schläpfer, S. Adriaensen, S. Kempenaers, D. Odermatt, M. Kneubuehler, J. Nieke, and K. Itten, "Structure, Components and Interfaces of the Airborne Prism Experiment (APEX) Processing and Archiving Facility," IEEE Transactions on Geoscience and Remote Sensing, vol. 47, pp. 29-43, 2009. 